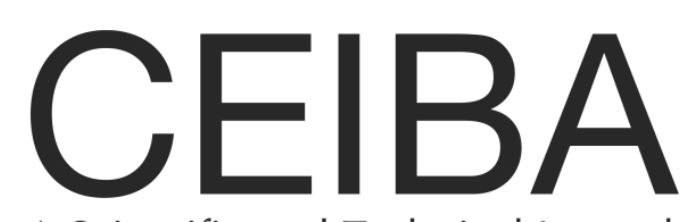

A Scientific and Technical Journal Published by Zamorano

\title{
0845
}

\section{Lyroglossa pubicaulis y Acianthera angustisepala: Nuevos registros de Orchidaceae para Honduras}

\author{
Dora Elisa Pérez \\ Universidad Nacional Autónoma de Honduras \\ Tegucigalpa, Honduras
}




\title{
Dora Elisa Pérez \\ Lyroglossa pubicaulis y Acianthera angustisepala: Nuevos registros de Orchidaceae para Honduras \\ Ceiba 0845: 1-2
}

DOI: $10.5377 /$ ceiba.v0i0845.9863

\author{
Publicado en el 2020 por \\ Escuela Agrícola Panamericana, Zamorano \\ P.O. Box 93 \\ Km 30 Tegucigalpa a Danlí, \\ San Antonio de Oriente, Francisco Morazán, Honduras
}

Ceiba es la revista científica y tecnológica de la Escuela Agrícola Panamericana, Zamorano, publicada desde 1950. A partir del 2019, Ceiba se publica exclusivamente de manera electrónica y en el formato de publicación continua; esto con el propósito de acortar el tiempo entre aceptación de un artículo y su publicación y que los autores puedan diseminar rápidamente los resultados de su investigación. Aceptamos artículos en español o en inglés relacionados con todas las áreas de la agricultura: agricultura tropical, recursos naturales, botánica, biología, ecología, desarrollo rural, forestales, economía agrícola, agronegocios, descripciones de nuevas especies, agroindustria, protección vegetal, notas científicas (plagas nuevas, observaciones interesantes) y resúmenes de tesis de los estudiantes de la Escuela Agrícola Panamericana. Ocasionalmente se publicarán revisiones bibliográficas y resúmenes de conferencias.

Los artículos para ser considerados para publicación tienen que ser originales y no deben haber sido publicados ni sometidos a otras revistas. Una vez sometidos a Ceiba tampoco deben someterse a otras revistas. Los autores deben indicar en su primer envío del artículo que cumplen con esta norma. Los artículos serán arbitrados por dos personas antes de aceptarlos para publicación usando el sistema de doble ciego. Ceiba usa revisores externos, el autor puede sugerir los revisores, pero no se garantiza que se usen. Después de ser revisado, los comentarios de los revisores se regresan al autor con el cual se trabaja en el manuscrito si este fue aceptado. Si el artículo es aceptado, se deben hacer las correcciones sugeridas por los revisores y regresarlo al editor en no más de dos semanas.

Los autores deben enviar sus artículos a través del sitio web único de la revista: https://www.lamjol.info/index.php/CEIBA para lo cual deben crear una cuenta en el sitio. Ceiba no dispone de ningún otro sitio para la recepción de manuscritos. En caso de algún problema, los autores pueden enviar por correo electrónico todos los archivos que forman el artículo (cuadros, fotografías, dibujos y gráficas) al editor, Dr. Jesús Orozco, al correo electrónico jorozco@zamorano.edu. Adicionalmente, pueden comunicarse con el editor al teléfono +504 2287-2000 ext 2340.

Ceiba está indexada en Web of Science, latindex y Central American Journals Online (CAMJOL).

Editor: Jesús Orozco

Diagramación: Jesús Orozco

Comité editorial: Ronald D. Cave, Mike D. Owen, José Manuel Mora, Carlos Hernández DíazAmbrona y Abelino Pitty

ISSN 2225-6687

Copyright. Este es un artículo de acceso abierto distribuido bajo los términos de Creative Commons, licencia de atribución no comercial, el cual permite el uso, distribución y reproducción no comercial en cualquier medio sin restricciones, siempre y cuando el autor original y la fuente sean acreditados. https://creativecommons.org/licenses/by-nc/3.0/deed.es. El copyright pertenece a los autores. 
Lyroglossa pubicaulis y Acianthera angustisepala: Nuevos registros de Orchidaceae para Honduras

\author{
Dora Elisa Pérez \\ doraelisa.perez53@gmail.com \\ Universidad Nacional Autónoma de Honduras \\ Tegucigalpa, Honduras
}

Resumen. Se registran dos especies de la familia Orchidaceae por primera vez para Honduras, Lyroglossa pubicaulis y Acianthera angustisepala. Con estos registros Honduras cuenta ahora con 680 orquídeas.

Palabras clave: Orquideoflora, diversidad, botánica

\title{
Lyroglossa pubicaulis and Acianthera angustisepala: New Orchidaceae records for Honduras
}

\begin{abstract}
Two new records are added to the orchid flora of Honduras: Lyroglossa pubicaulis and Acianthera angustisepala. With these new record Honduras has now 680 orchids known.
\end{abstract}

Key words: Orchid flora, diversity, botany.

Honduras cuenta con alrededor de 680 especies de orquídeas; esta cifra se alcanza sumando a las 652 de Nelson (2008, 2010) las registradas por Vega et. al. (2014, 2016), Linares y Ancalmo (2016), Pérez (2017), y las incluidas en este trabajo.

Para la obtención de los especímenes se hizo un recorrido por el área de amortiguamiento del Parque Nacional La Tigra (Francisco Morazán), donde se encontraron unos pocos ejemplares de la orquídea terrestre Lyroglossa pubicaulis. Se tomaron los datos para su descripción y se depositó un ejemplar en el herbario Paul C. Standley (EAP), Escuela Agrícola Panamericana. El ejemplar de Acianthera angustisepala se identificó de los ejemplares cultivados por la autora provenientes de Comayagua. Se tomaron fotos y se hizo la descripción respectiva. Actualmente el ejemplar se encuentra depositado en el orquideario de Jorge Bueso Arias (Escuela Agrícola Panamericana).

Lyroglossa pubicaulis (L.O. Williams) Garay: Distribución: Belice, El Caribe, Guatemala, México, Nicaragua (Tropicos.org 2020a), y Honduras. Descripción: Hierba terrestre erecta de flores verdes, no fragantes, densamente pubescentes, labelo blanco, con venas verde oscuro, Fig. 1A. Hábitat en Honduras: Bosque de pino abierto, mezclado con gramínea, $1300 \mathrm{~m}$. Fenología: florece de diciembre a enero.

Material examinado: Francisco Morazán: Aldea La Unión, Municipio de Santa Lucía, área de amortiguamiento Parque Nacional la Tigra, 22 de diciembre 2018, 1300 m, D.E. Pérez 733 (EAP).

Acianthera angustisepala (Ames y Correll) Pridgeon y M.W. Chase. Distribución: México, Guatemala, Belice (Tropicos.org 2020b), y Honduras. Descripción: Hierba epífita pequeña, inflorescencia apical, flores amarillo-verdoso con manchas café-púrpura, Fig. 1B. Hábitat en Honduras: Bosque nublado. Fenología: florece de agosto a octubre.

Material examinado: Comprada en Siguatepeque, Comayagua, 2015, Dora Pérez s.n.

\section{Agradecimientos}

La autora agradece a Cyril H. Nelson por los comentarios y revisión al manuscrito y a Rina Fabiola Díaz por la preparación del material para el herbario. 

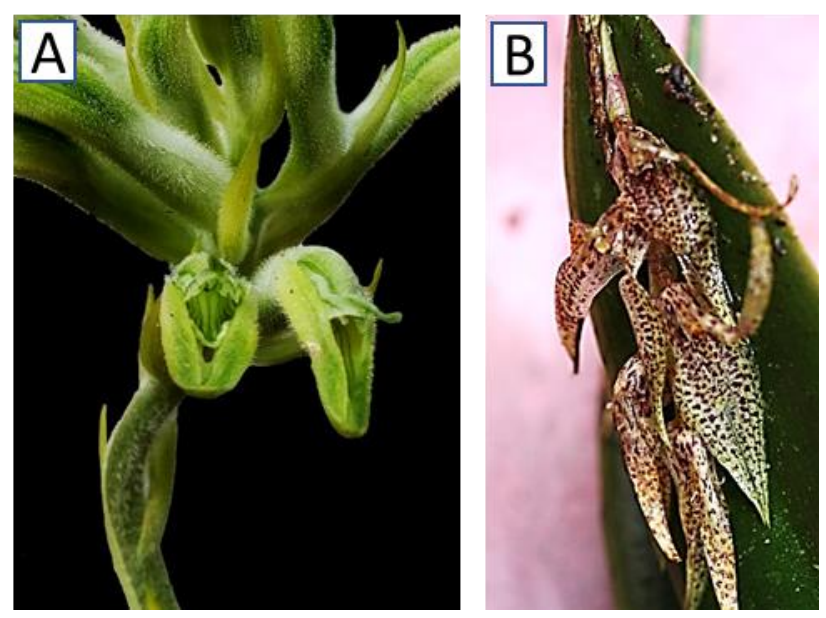

Figura 1. Nuevas orquídeas de Honduras. A) Lyroglossa pubicaulis (L.O. Williams) Garay; B) Acianthera angustisepala (Ames \& Correll) Pridgeon \& M.W. Chase.

\section{Literatura Citada}

Linares JL, Ancalmo P. 2016. Una nueva especie de Sobralia (Orchidaceae) de Honduras. Journal of the Botanical Research Institute of Texas. 10(1):77 -83.

Nelson CH. 2008. Catálogo de las plantas vasculares de Honduras. Secretaría de Recursos naturales y Ambiente, Tegucigalpa, Honduras. Tegucigalpa, 1576 p.

Nelson CH. 2010. Adiciones y comentarios a la flora de Honduras. Ceiba .51(2):70 -88.

Pérez DE. 2017. Cuatro nuevos registros de Orchidaceae para Honduras. Lankesteriana. 17(3):411 -415.

Tropicos.org. 2020a. Missouri Botanical Garden. http://www.tropicos.org/Name/23515544 Accesado: $26 / 06 / 2020$

Tropicos.org. 2020b. Missouri Botanical Garden. http://www.tropicos.org/Name/50210009 Accesado $26 / 06 / 2020$

Vega H, Cetzal-Ix W, Mo E, Germer D, Soler K. 2014. Nuevos registros de Orchidaceae para Honduras. Lankesteriana. 14(2):115-121.

Vega H, Cetzal-Ix W, Mo E, Romero-Soler K. 2016. Nuevos registros para la flora de Honduras y el Parque Nacional Montaña de Celaque. Acta Biológica Colombiana. 21(3):635 -644.

Recibido marzo 13, 2020; aceptado junio 10, 2020.

Cómo citar: Pérez DE. 2020. Lyroglossa pubicaulis y Acianthera angustisepala: Nuevos registros de Orchidaceae para Honduras. Ceiba. 0845:1-2. 